\title{
Predisposing Sinonasal Disease Foci: Do They Really Affect Mucosal Chronic Otitis Media?
}

\author{
Vikram Kemmannu Bhat ${ }^{1}$ Aroosh Thevaruparambil Paramu ${ }^{2}$ Shwetha Nagachar ${ }^{1}$ \\ ${ }^{1}$ Department of Otolaryngology, Head and Neck Surgery, Karnataka \\ Institute of Medical Sciences, Hubli, Karnataka, India \\ 2Department of ENT, Sacred Heart General (SHG) Hospital, \\ Alappuzha District, Kerala, India \\ Address for correspondence Vikram Kemmannu Bhat, MS, DNB, \\ MNAMS, PhD, Department of Otolaryngology, Head and Neck \\ Surgery, Karnataka Institute of Medical Sciences, Vidyanagar, \\ Hubli 580021, Karnataka, India (e-mail: vikram.ent@gmail.com).
}

Ann Otol Neurotol ISO 2018;1:100-104

\begin{abstract}
Background This study aimed to find the relationship between sinonasal predisposing foci in chronic otitis media mucosal disease (COMMD) and risk from these predisposing disease foci, and to identify the correlation of bacterial flora of the ear and nose in chronic otitis media.

Materials and Methods This was a prospective case-control study with 100 cases and 100 controls. All patients underwent a detailed clinical ear-nose-throat (ENT) examination and diagnostic nasal endoscopy to look for sinonasal and pharyngeal pathology. In all patients, ear and nasal swabs were taken at the first visit. The relationship between these factors and COMMD was studied by appropriate statistical tests.

Observations and Results Extremely significant association between tubal tonsil hypertrophy and COMMD, very significant association between adenoid hypertrophy and COMMD, and just significant association between deviated nasal septum, inferior turbinate hypertrophy, concha bullosa, and COMMD were found. No significant association was found between sinusitis and COMMD. In this study Staphylococcus aureus (51\%) was the predominant organism isolated from ear swab, followed by Pseudomonas (27\%). The same organism in nasal and ear swabs was demonstrated only in eight (13\%) cases. Conclusion Sinonasal predisposing foci play a substantial role in the development of

Keywords

- otitis media

- chronic disease

- Eustachian tube

- nose

- throat COMMD and observed in $97 \%$ of the cases with bilateral disease and $91 \%$ with unilateral disease in this study. Hence, every case of COMMD must undergo investigations such as diagnostic nasal endoscopy to discover these foci. Because sinonasal disease is one of the important factors contributing to otitis media, timely detection and management of symptomatic sinonasal disease must precede surgical management of COMMD.
\end{abstract}

\section{Introduction}

Chronic otitis media is a widespread disease of the developing countries, especially the rural areas. The etiology and pathogenesis of chronic otitis media mucosal type (COMMD) are multifactorial. The more relevant factor in the evolution of this disorder is the Eustachian tube dysfunction. The sinonasal predisposing disease foci present as extrinsic factors to influence the function of the Eustachian tube. However, their precise role and influence on the latter are one of the many gray areas in the pathogenesis of otitis media. Even the duration of influence of these foci on the middle ear function is a matter of controversy. In few cases, dormant foci in the nose and throat can become clinically significant after surgery and lead to recurrence of ear disease and failure of ear surgery. ${ }^{1}$

Preoperative evaluation of the predisposing focus in the nose and throat in COMMD will be helpful in determining the potential results of tympanoplasty and mastoid surgery and hence the need for study. 


\section{Materials and Methods}

This study was conducted in the department of earnose-throat (ENT) in a tertiary care public hospital. Ethical clearance from institutional review board was obtained. Patients in the age group of 14 to 70 years attending the outpatient clinic of the department in a span of 1 year were recruited into the study. Equal number of age and sex matched controls were also recruited. This was a prospective case-control study with two arms. The cases included the patients having COMMD without complications. The controls were those patients who had no symptoms and signs of ear disease. Patients with acute otitis media, adhesive otitis media, and otitis media with effusion, chronic otitis media squamosal type, or tympanic perforation of traumatic etiology were excluded.

Every consecutive eligible patient was recruited into the study. All patients underwent a detailed clinical ENT examination. They were subjected to rigid diagnostic nasal endoscopy (DNE) and findings noted in each case with respect to adenoid enlargement, tubal tonsil hypertrophy, movement of eustachian tube pharyngeal opening, inferior turbinate hypertrophy, bullous concha, deviated nasal septum, discharge in middle meatus, nasal polyp, atrophic rhinitis, etc. One hundred controls were also recruited at random who were age and sex matched with the cases. DNE was also performed in controls to search for the same foci. Both cases and controls were subjected to other investigations when needed such as computed tomographic (CT) scan of the nose and paranasal sinuses only after DNE.

In all cases, ear and nasal swabs were taken at the first visit for microbiological analysis. The pathology in the nose and throat and its side in relation to COMMD was the primary outcome. Bacterial flora of the nose and ear was the secondary outcome.

\section{Observations and Results}

A total of 100 patients with COMMD and 100 age and sex matched controls were recruited into the study. There were 34 cases of right-sided, 34 cases of left-sided, and 32 cases of bilateral COMMD.

\section{Age Distribution in Study Population}

In this study, presence of predisposing disease foci was evaluated in patients in the age group of 14 to 70 years. The youngest patient was 14 years, and the oldest was 67 years in cases, and the youngest and oldest patients in control group were 14 years and 65 years, respectively. Mean age of the case and control groups were 26.41 and 29.21 , respectively. There was no significant difference between the two groups in age distribution.

\section{Predisposing Foci and Chronic Otitis Media Mucosal Disease}

In our study, $93 \%$ of cases and $73 \%$ of controls had predisposing foci. Out of 32 bilateral COMMD cases, only one case had no predisposing foci. Symptomatic predisposing foci were present in $52 \%$ of cases and $55 \%$ of controls (-Table 1). There was extremely significant association between predisposing factors and COMMD. There was 4.9 times increased risk of developing COMMD in the presence of predisposing foci.

\section{Single and Multiple Foci in Cases and Controls}

In this study, $82 \%$ of cases had multiple foci compared with $65 \%$ in controls. The association was significant. There was 2.5 times increased risk of developing COMMD in the presence of multiple foci (-Table 2). - Table 3 compares the risk of COMMD with increase in number of foci. With increase in number of foci, odds ratio increased, which suggested the increasing risk of developing COMMD. - Table 4 compares

Table 1 Comparison of cases and controls with respect to predisposing foci

\begin{tabular}{|l|l|l|l|}
\hline Status & Cases & Controls & Total \\
\hline Predisposing focus present & 93 & 73 & 166 \\
\hline Predisposing focus absent & 7 & 27 & 34 \\
\hline Total & 100 & 100 & 200 \\
\hline
\end{tabular}

Chi-square $=14.1743, p=0.0000, p<0.05$.

Odds ratio $=4.91,95 \% \mathrm{Cl}=2.05-11.78$.

Table 2 Comparison of single and multiple foci in cases and controls

\begin{tabular}{|l|l|l|l|l|}
\hline $\begin{array}{l}\text { Foci in } \\
\text { nose } \\
\text { and } \\
\text { throat }\end{array}$ & $\begin{array}{l}\text { Cases } \\
\text { with foci } \\
(\boldsymbol{n}=\mathbf{9 3})\end{array}$ & Percentage & $\begin{array}{l}\text { Controls } \\
\text { with } \\
\text { foci } \\
(\boldsymbol{n}=\mathbf{7 3})\end{array}$ & Percentage \\
\hline $\begin{array}{l}\text { Single } \\
\text { focus }\end{array}$ & 16 & 17.20 & 25 & 34.25 \\
\hline $\begin{array}{l}\text { Multiple } \\
\text { foci }\end{array}$ & 77 & 82.80 & 48 & 65.75 \\
\hline
\end{tabular}

Chi-square $=6.3872, p=0.0121, p<0.05$.

Odds ratio $=2.51,95 \% \mathrm{Cl}=1.22-5.15$.

Table 3 The odds ratio for the number of sinonasal foci

\begin{tabular}{|l|l|}
\hline Number of foci & Odds ratio \\
\hline One & 2.46 \\
\hline Two & 3.65 \\
\hline Three & 11.95 \\
\hline More than three & 42 \\
\hline
\end{tabular}

Table 4 Comparison of bilateral and unilateral COMMD in relation to the presence or absence of sinonasal focus

\begin{tabular}{|l|l|l|l|l|}
\hline & $\begin{array}{l}\text { Cases } \\
\text { with } \\
\text { foci } \\
(\boldsymbol{n}=\mathbf{9 3})\end{array}$ & Percentage & $\begin{array}{l}\text { Cases } \\
\text { without } \\
\text { focus } \\
(\boldsymbol{n}=7)\end{array}$ & Percentage \\
\hline $\begin{array}{l}\text { Bilateral } \\
\text { COMMD }\end{array}$ & 31 & 33.33 & 1 & 14.28 \\
\hline $\begin{array}{l}\text { Unilateral } \\
\text { COMMD }\end{array}$ & 62 & 66.66 & 6 & 85.71 \\
\hline
\end{tabular}

Abbreviation: COMMD, chronic otitis media mucosal disease. Chi-square $=1.0854, p=0.2974, p<0.05$.

Odds ratio $=3$. 
Table 5 Comparison of foci in cases and controls

\begin{tabular}{|l|l|l|l|l|l|l|}
\hline & Adenoid enlargement & Tubal tonsil & Sinusitis & DNS & ITH & Bullous concha \\
\hline Cases & $\begin{array}{l}34 \% \\
(58 \%)\end{array}$ & $39 \%$ & $14 \%$ & $\begin{array}{l}77 \% \\
(48 \%)\end{array}$ & $\begin{array}{l}47 \% \\
(45 \%)\end{array}$ & $13 \%$ \\
\hline Controls & $\begin{array}{l}16 \% \\
(48 \%)\end{array}$ & $6 \%$ & $12 \%$ & $\begin{array}{l}63 \% \\
(52 \%)\end{array}$ & $\begin{array}{l}32 \% \\
(40 \%)\end{array}$ & $5 \%$ \\
\hline$p$-Value & 0.0030 & 0.000 & 0.674 & 0.031 & 0.003 & 0.0482 \\
\hline Odds ratio & 2.70 & 10.02 & 1.19 & 1.97 & 1.88 & 2.84 \\
\hline
\end{tabular}

Abbreviations: DNS, deviated nasal septum; ITH, inferior turbinate hypertrophy.

the association of bilateral and unilateral COMMD with and without sinonasal focus. There was no significant difference in their association even though the chance of finding a focus was three times more in bilateral COMMD as compared with unilateral COMMD. Association between the various sinonasal pathologies and COMMD and also the risk factor in their presence are given in - Table $\mathbf{5}$. The percentages within brackets indicate the patients who had symptoms linked to the focus.

\section{Side of Deviated Nasal Septum, Inferior Turbinate Hypertrophy, and Side of Chronic Otitis Media Mucosal Disease}

In this study, 26 patients with right-sided, 24 with left-sided, and 27 with bilateral COMMD had deviated nasal septum. In patients with right COMMD, $46 \%$ had right deviated nasal septum (DNS); in left COMMD cases, 50\% had left DNS; and in bilateral cases, 40\% had right DNS and 33\% had left DNS. Even though right COMMD cases with right DNS and left COMMD cases with left DNS were more, the association was not statistically significant.

The side of COMMD was compared with the side of inferior turbinate hypertrophy (ITH). No significant association was found between side of COMMD and side of ITH.

\section{Microbiology of Ear and Nasal Swabs}

Out of 100 patients examined, positive ear swab was obtained in 59 ears and positive nasal swab was obtained in 20 cases (-Table 6). In 8 (13\%) cases, same organism was found in both nasal and ear swabs. Number of organisms isolated from ear and nasal swabs were 64 and 22, respectively. Staphylococcus aureus was the most common organism isolated from both ear (51\%) and nose (65\%), followed by Pseudomonas (27\% in the ear and $20 \%$ in the nose). More than one organism was isolated in four ear swabs and two nasal swabs.

Table 6 Microbiological organisms found in ear and nasal swabs

\begin{tabular}{|l|l|l|}
\hline Organism & Ear swab & Nasal swab \\
\hline Staphylococcus aureus & 38 & 16 \\
\hline Pseudomonas & 16 & 4 \\
\hline Klebsiella & 4 & 1 \\
\hline Proteus & 2 & 1 \\
\hline Escherichia coli & 3 & 0 \\
\hline $\begin{array}{l}\text { Nonfermenting } \\
\text { gram-negative bacilli }\end{array}$ & 1 & 0 \\
\hline No organism & 73 & 112 \\
\hline
\end{tabular}

\section{Discussion}

COMMD is one of the most common causes of conductive hearing loss in the tropical developing nations. The most important factor in the evolution of this disorder is supposed to be the Eustachian tube dysfunction. Patients with chronic ear disease frequently have coexistent sinonasal pathology, which can trigger the middle ear problems secondary to dysfunction of the Eustachian tube.

The relationship between sinonasal disease foci and COMMD is complicated, and even today it remains poorly understood. This understanding is crucial not only for the cure of middle ear disease but also for the prevention of its recurrence. Unfortunately, the demarcation between anatomical variation and pathological disease is narrow and poorly defined in many structures in the sinonasal region. What constitutes an active pathological focus in the nose with regard to DNS, concha bullosa, and a few other anatomical variations is difficult to identify. These so-called anatomical variations in the nose can become pathological at times or indirectly contribute to some other pathology and hence transform into an actual "contributing" predisposing focus. However, the remedial surgery for these foci is controversial and justified mostly in the presence of symptoms linked to the focus. Foci in the nose and throat can become active and inactive at various times. Hence, they may not appear and present as a disease focus at the point of time of DNE. Even though a disease focus may not appear to be active and pathological at the time of DNE, it could have been active enough in the past to have triggered and sustained suppurative otitis media up to the point of chronic perforation syndrome.

In our own study ${ }^{2}$ comprising 187 ears with complicated and uncomplicated COMMD, a contributing disease focus in the nose or throat was found in $70.96 \%$ of complicated cases and in $84 \%$ of uncomplicated cases, but its presence did not increase the risk for complications.

In yet another study ${ }^{1}$ of ours involving 68 patients with COMMD, we found that most of surgical failures in tympanoplasty had a reactivated, predisposing focus in the nose and throat that included persistent adenoid, sinusitis, nasal polyp, allergic rhinitis, and atrophic rhinitis.

The covariation between ear infection and tonsillitis, sinusitis, and atopic diseases was studied in 1996 by Kvaerner et $\mathrm{al}^{3}$ They found a clustering tendency among the upper respiratory tract infections.

In 2011 Yeolekar and Dasgupta ${ }^{4}$ studied the influence of sinonasal disease on the middle ear condition. Out of 
100 COMMD cases, 80 had DNS. Significant improvement of middle ear disease was found in $84.53 \%$ ears after septal correction, $82.35 \%$ ears after treatment of sinusitis, and $76.92 \%$ ears after polypectomy.

Improvement of middle ear pathology following septoplasty was noted by Grady et $\mathrm{al}^{5}$ in 1983 . Of the 75 septoplasty cases reviewed, $13 \%$ had associated middle ear pathology, and 70\% among these patients showed significant improvement of their ear disease following septoplasty.

In 1983 Van Cauwenberge et $\mathrm{al}^{6}$ studied the relationship between nasal and middle ear pathology in school going children. They found a well-defined influence of septal deviation on middle ear status.

In our study, significant association was found between DNS and COMMD. Seventy seven percent of cases and $63 \%$ of controls had DNS that was comparable with the above studies. In the presence of DNS, there was two times increased risk of developing COMMD. Significant association was also found between ITH, bullous concha, and COMMD.

Güçlü et $\mathrm{al}^{7}$ evaluated nasal airways by objective methods in chronic otitis media. They found significantly higher nasal airway resistance in chronic otitis media cases than control group. There was no statistical difference between the measurements on each side in unilateral chronic otitis media.

In our study, no significant association was found between sides of COMMD and DNS or side of ITH. DNS does not cause Eustachian tube dysfunction due to mechanical obstruction, but it causes pressure changes in nasopharynx and causes functional obstruction. As the nasopharynx is one cavity, whichever side the septum is deviated, it will cause pressure changes in nasopharynx and can cause disease in either ear. Other mechanism may be the compensatory ITH on the side opposite to DNS. In our study 77 patients had DNS, out of whom 43 (56\%) had ITH.

Singh and Arora ${ }^{8}$ in 1977 in India studied 100 patients in the age group 4 to 16 years, who were divided into three groups: 25 normal patients without any adenoid hypertrophy symptoms or ear discharge, 25 patients with adenoid hypertrophy symptoms, and 50 patients with COMMD. They compared the mean adenoid mass area, mean bony nasopharynx, and the ratio between these areas. They found that in adenoid hypertrophy with COMMD, the ratio of adenoid mass area to the bony nasopharynx was more than $50 \%$ and concluded that adenoids were etiological in COMMD and advocated their removal. de Aquino et $\mathrm{al}^{9}$ in 2007 studied 30 patients with chronic otitis media. Nasal endoscopy was done to look for the pharyngeal end of the Eustachian tube. They found that $33 \%$ had modified pharyngeal ostium, and among the modified ostiums, 19\% had tubal tonsil hypertrophy.

In our study $39 \%$ of cases and $6 \%$ controls had tubal tonsil hypertrophy, and the association was found to be extremely significant. In presence of tubal tonsil hypertrophy, there was 10 times increased risk of developing COMMD. The location of the tubal tonsils logically suggests that a potential inflammation obstructs the proximal end of the Eustachian tube and in turn causes negative middle ear pressure and influx of nasopharyngeal secretion into the middle ear.
No significant association was found between sinusitis and COMMD, not in agreement with other studies like Brook et $\mathrm{al}^{10}$ and Mills et $\mathrm{al} .{ }^{11}$ It may be because our study population had less cases of sinusitis and it was equally present in controls too.

When one, two, and three foci were compared with no focus ( - Table 3 ), there was an increase in odds ratio with an increase in the number of foci (dose response relationship), which probably hinted toward causation. The other findings in this study that supported causation included

1. Very few cases of COMMD did not have any sinonasal predisposing disease focus. This was truer with bilateral COMMD. There was only one case of bilateral COMMD without a focus.

2. There was a temporal relationship between presence of focus and development of COMMD.

3. Very strong association existed between certain foci such as tubal tonsil hypertrophy, adenoid hypertrophy, and COMMD.

Though many previous studies by Yeolekar et $\mathrm{al}^{4}$ and Grandy et $\mathrm{al}^{5}$ concluded that surgical correction of DNS improved middle ear disease, none of studies mentioned whether it was isolated DNS or with other risk factors.

Rao and Reddy ${ }^{12}$ in 1994 studied 120 cases of chronic suppurative otitis media and found S. aureus (42.5\%) as the predominant organism, followed by Pseudomonas (21\%) and Proteus species (18.33\%). A single pathogen was isolated from $68.52 \%$ cases and mixed growth from the rest.

Baruah et $\mathrm{al}^{13}$ in 1972 in their study found out that the predominant organism was S. aureus (33.98\%), followed by Pseudomonas (25\%) and Proteus species. They compared the nasal and ear swabs and concluded that correlation between the organism present in the nasopharynx and throat was much better in acute suppurative otitis media and poor (15\%) in chronic suppurative otitis media.

Grewal et $\mathrm{al}^{14}$ in 1982 studied 50 cases of chronic suppurative otitis media. Bacteriological study of the nasopharynx and ear was done and compared. They could not demonstrate any appreciable agreement between the organisms isolated from the nasopharynx and ear (6\%).

In this study too, no agreement was found between flora of the nose and ear. Same organism in nasal and ear swabs was demonstrated in only eight (13\%) ears. These results were comparable with the above studies.

\section{Conclusion}

Sinonasal predisposing foci play a complex role in the pathogenesis of COMMD. A significant proportion of these foci remain clinically silent and undiagnosed. Also, most (91\%) of the COMMD cases with unilateral disease have some or the other predisposing focus and more so (97\%) in cases with bilateral disease. As the number of foci increases, the risk of developing COMMD also increases substantially. The chance of finding a potential sinonasal focus is much more in bilateral than unilateral COMMD. There is a 
significant association between tubal tonsil hypertrophy, adenoid hypertrophy, DNS, ITH, bullous concha, and COMMD. They also pose a risk factor for the pathogenesis of COMMD. Hence, every case of COMMD must undergo investigations such as DNE to discover these foci. There is no association between the sides of focus (DNS and ITH) and of COMMD. The flora in the sinonasal region and COMMD are not the same in most cases. In the background of all these revelations, we recommend that symptomatic sinonasal foci be adequately treated before surgically treating COMMD.

\section{Conflict of Interest}

None.

\section{References}

1 Bhat KV, Naseeruddin K, Nagalotimath US, Kumar PR, Hegde JS. Cortical mastoidectomy in quiescent, tubotympanic, chronic otitis media: is it routinely necessary? J Laryngol Otol 2009;123(4):383-390

2 Vikram BK, Khaja N, Udayashankar SG, Venkatesha BK, Manjunath D. Clinico-epidemiological study of complicated and uncomplicated chronic suppurative otitis media. J Laryngol Otol 2008;122(5):442-446

3 Kvaerner KJ, Tambs K, Harris JR, Mair IW, Magnus P. Otitis media: relationship to tonsillitis, sinusitis and atopic diseases. Int J Pediatr Otorhinolaryngol 1996;35(2):127-141

4 Yeolekar AM, Dasgupta KS. Otitis media: does the onus lie on sinonasal pathology? Indian J Otol 2011;17(1):8-11
5 Grady D, Mathias P, Anderson R, Snider G, Sprinkle PM. Improvement of middle ear disease following septoplasty. Am J Otol 1983;4(4):327-331

6 Van Cauwenberge P, Derycke A. The relationship between nasal and middle ear pathology. Acta Otorhinolaryngol Belg 1983;37(6):830-841

7 Güçlü O, Sahin EM, Tekin K, Dereköy FS. Evaluation of nasal airways by objective methods in chronic otitis media. Eur Arch Otorhinolaryngol 2013;270(4):1263-1266

8 Singh A, Arora MML. Relationship between adenoids and chronic suppurative otitis media. Indian J Otolaryngol Head Neck Surg 1977;29(2):69-71

9 de Aquino JEAP, Zavarezzi DE, Carvalho MRMS, Aquino JNP. Endoscopic evaluation of pharyngeal orifice of eustachian tube in patients with chronic otitis. Int Arch Otorhinolaryngol 2007;11(2):106-108

10 Brook I, Yocum P, Shah K. Aerobic and anaerobic bacteriology of concurrent chronic otitis media with effusion and chronic sinusitis in children. Arch Otolaryngol Head Neck Surg 2000;126(2):174-176

11 Mills RP, Irani BS, Vaughan-Jones RJ, Padgham ND. Maxillary sinusitis in children with otitis media with effusion. J Laryngol Otol 1994;108(10):842-844

12 Rao BN, Reddy MS. Chronic suppurative otitis media-a prospective study. Indian J Otolaryngol Head Neck Surg 1994;46(2):72-77

13 Baruah PC, Agarwal SC, Arora MML, Mehra YN. Clinical and microbiological studies in suppurative otitis media in Chandigarh. Indian J Otolaryngol Head Neck Surg 1972;24(4):157-160

14 Grewal DS, Hiranandani NL, Pusalkar AG. The middle ear mucosa in chronic suppurative otitis media. Indian J Otolaryngol Head Neck Surg 1982;34(2):1-5 